\title{
QUALIDADE DE VIDA DE HIPERTENSOS EM TRATAMENTO AMBULATORIAL
}

\author{
Natália Carolina Mian¹, Renata Cristina Gasparino²
}

RESUMO: Pesquisa descritiva e transversal que teve como objetivo identificar a qualidade de vida de pacientes hipertensos em tratamento ambulatorial. Participaram 88 pacientes hipertensos, a maioria mulheres, atendidos num ambulatório de especialidades do interior do Estado de São Paulo. Para a coleta de dados foram utilizados: uma ficha de caracterização sociodemográfica e o Mini-Cuestionario de Calidad de Vida en Hiperténsion Arterial, adaptado e validado para a cultura brasileira. A maioria dos pacientes alegou não ser tabagista, não fazer uso de bebida alcoólica e não praticar exercício físico. A média das respostas revelou pior percepção da qualidade de vida em comparação a outros estudos. Esta pesquisa auxilia a equipe de saúde a propor intervenções para o controle da hipertensão que minimizem o comprometimento da qualidade de vida dos portadores.

PALAVRAS-CHAVE: Qualidade de vida; Hipertensão; Pacientes ambulatoriais.

\section{QUALITY OF LIFE IN HYPERTENSIVES RECEIVING OUTPATIENT TREATMENT}

ABSTRACT: This descriptive, cross-sectional research aimed to identify the quality of life among hypertensive patients receiving outpatient treatment. Eighty-eight predominantly female hypertensive patients participated, attended in an outpatient clinic in the state of São Paulo. The following were used for collecting data: a form on sociodemographic characterization and the Mini-Cuestionario de Calidad de Vida en Hiperténsion Arterial, adapted and validated for Brazilian culture. The majority of the patients claimed to be non-smokers, not to use alcohol and not to practise physical exercise. The average of the responses showed a worse perception of quality of life in comparison with other studies. This research helped the health team to propose interventions for controlling hypertension which minimize the compromising of quality of life among hypertensives.

KEYWORDS: Quality of life; Hypertension; Outpatients.

\section{CUALIDAD DE VIDA DE HIPERTENSOS EN TRATAMIENTO EN AMBULATORIO}

RESUMEN: Investigación descriptiva y transversal que tuvo como objetivo identificar la cualidad de vida de pacientes hipertensos en tratamiento en ambulatorio. Participaron 88 pacientes hipertensos, la mayoría mujeres, atendidos en un ambulatorio de especialidades del interior del estado de São Paulo. Para obtener los datos, fueron utilizados: una ficha de caracterización sociodemográfica y el Minicuestionario de Cualidad de Vida en Hiperténsion Arterial, adaptado y validado para la cultura brasileña. La mayoría de los pacientes alegó no ser tabaquista, no hacer uso de bebida alcohólica y no practicar ejercicio físico. La media de las respuestas ha revelado peor percepción de la cualidad de vida en comparación a otros estudios. Esta investigación ayuda el equipo de salud a proponer intervenciones para el control de la hipertension que minimizen el comprometimiento de la cualidad de vida de los portadores.

PALABRAS CLAVES: Cualidad de vida; Hipertensión; Pacientes de ambulatorio.

${ }^{1}$ Enfermeira. Especializanda em Cardiologia pelo Instituto do Coração, Jundiaí - SP

${ }^{2}$ Enfermeira. Mestre em Enfermagem. Doutoranda pelo Programa de Pós-Graduação em Enfermagem da Universidade Estadual de Campinas. Professora da Faculdade de Medicina de Jundiaí - SP. 


\section{INTRODUÇÃO}

Hipertensão arterial sistêmica (HAS) é uma condição clínica na qual o paciente apresenta níveis de pressão arterial (PA) acima dos parâmetros considerados normais. A HAS associada, ou não, a outros fatores de risco, pode levar o indivíduo a desenvolver diversas complicações como doença cerebrovascular, doença arterial coronariana, insuficiência cardíaca, insuficiência renal crônica e doença vascular de extremidades ${ }^{(1)}$.

Para estabelecer o diagnóstico e tratamento apropriados devem ser considerados os aspectos clínicos, laboratoriais e os valores de PA. Os objetivos dessa avaliação são identificar fatores de risco para doenças cardiovasculares, pesquisar lesões de órgãos-alvo, identificar a presença de outras doenças associadas, estratificar o risco cardiovascular global e avaliar indícios de hipertensão arterial secundária ${ }^{(1)}$.

A terapêutica medicamentosa adotada deve ser individualizada e estar de acordo com o risco que o paciente apresenta, porém o que se preconiza para todos os pacientes, independentemente do risco cardiovascular, é a mudança do estilo de vida e hábitos alimentares, ou seja, o estabelecimento de um tratamento não medicamentoso ${ }^{(2)}$.

É nesse contexto que a equipe multiprofissional deve agir para que o paciente tenha uma visão ampla do seu estado de saúde e possa compreender melhor os riscos que corre se não aderir ao tratamento. $\mathrm{O}$ trabalho de uma equipe multidisciplinar gera melhores resultados na mudança dos fatores reversíveis (controle de peso, consumo de sal, ingestão de bebidas alcoólicas, estresse, abandono do tabagismo e sedentarismo) que levam a $\mathrm{HAS}^{(1,3)}$.

O uso contínuo de medicações e a mudança dos hábitos de vida, com o intuito de controlar os fatores de risco reversíveis, podem refletir diretamente na adesão ao tratamento e, consequentemente, na qualidade de vida desses portadores. Qualidade de vida pode ser definida como a percepção do indivíduo de sua posição no contexto da cultura e sistema de valores nos quais ele vive e em relação aos seus objetivos, expectativas, padrões e preocupações ${ }^{(4)}$.

Os profissionais da área da saúde preocupam-se, cada vez mais, com a preservação da qualidade de vida dos pacientes por meio da prevenção ou tratamento das enfermidades, prova disso é o aumento no número de publicações que fazem referência a esse tema nos últimos $\operatorname{anos}^{(5)}$. Identificar a qualidade de vida dos indivíduos com HAS significa compreender suas ne- cessidades e propor intervenções que comprometam, o mínimo possível, a mudança dos hábitos de vida e a adesão ao tratamento. Portanto, o objetivo do presente estudo foi identificar a qualidade de vida de pacientes hipertensos em tratamento ambulatorial.

\section{MÉTODO}

Estudo descritivo e transversal realizado no Ambulatório de Especialidades da Faculdade de Medicina de Jundiaí, interior do Estado de São Paulo. Este ambulatório está em funcionamento desde 1997 e lá atuam alunos e docentes no atendimento a pacientes encaminhados de unidades básicas de Jundiaí e região. A amostra foi composta por 88 pacientes que atenderam aos seguintes critérios de inclusão: ser portador de HAS, ter idade igual ou superior a 18 anos, e estar em tratamento ambulatorial.

Os participantes, após aceitarem e formalizarem a participação no estudo, responderam uma ficha de caracterização sociodemográfica e o instrumento Mini - Cuestionario de Calidad Vida en Hiperténsion Arterial (MINICHAL). A ficha de caracterização sociodemográfica e clínica abordou dados como: idade, sexo, raça, estado civil, renda familiar, grau de escolaridade, hábitos como tabagismo e ingestão de bebida alcoólica, atividade física, tempo de tratamento da HAS, presença de co-morbidades e complicações.

O MINICHAL tem por objetivo identificar a percepção dos pacientes hipertensos sobre sua qualidade de vida. O miniquestionário de qualidade de vida em hipertensão arterial - Brasil (MINICHAL - BRASIL) foi adaptado e validado para a cultura brasileira e escolhido para este estudo por ser de rápida e fácil aplicação e possuir propriedades psicométricas consideradas satisfatórias ${ }^{(5)}$.

O MINICHAL - BRASIL é composto por 17 itens divididos em duas subescalas: estado mental que avalia o nível de ansiedade e depressão dos pacientes e é composta por nove itens e manifestações somáticas, composta por sete itens, que avalia a sensação de estar doente. A questão de número 17 avalia como o paciente percebe que a HAS e seu tratamento têm influenciado sua qualidade de vida. O participante é solicitado a responder as questões fazendo referência aos últimos sete dias ${ }^{(5)}$.

A escala de medida utilizada é a do tipo Likert que varia entre zero (não, absolutamente) e três pontos (sim, muito), assim, quanto mais próxima de zero for a pontuação, melhor a qualidade de vida. Os escores são obtidos pela soma das respostas dos sujeitos para 
cada subescala, podendo variar entre zero e 27 pontos para a subescala estado mental e entre zero e 21 para a subescala manifestações somáticas ${ }^{(5)}$.

Os dados foram coletados no período de abril a julho de 2010, nos dias em que os cardiologistas possuíam agenda para atendimento. Os sujeitos foram rastreados no momento de abertura da ficha de atendimento, na recepção e aqueles que atenderam aos critérios de inclusão foram convidados a participar da pesquisa.

A pesquisa obteve parecer favorável do Comitê de Ética em Pesquisa da Faculdade de Medicina de Jundiaí, protocolo n. 28/10 e aprovação da coordenação do ambulatório onde o estudo foi realizado. A pesquisa foi desenvolvida de acordo com as normas de pesquisa envolvendo seres humanos, Resolução do Conselho Nacional de Saúde 196/96 ${ }^{(6)}$ e não apresentou quaisquer riscos de danos à dimensão física, psíquica, moral, intelectual, social, cultural ou espiritual dos respondentes.

Os dados foram tabulados no programa Excel - Windows/XP e analisados por um estatístico por meio do programa Statistical Analysis System (SAS System for Windows), versão 9.1. Foi utilizada análise descritiva com elaboração de tabelas de frequência das variáveis categóricas e medidas de posição das variáveis contínuas. Os escores dos domínios foram obtidos pela soma das respostas dos sujeitos.

A confiabilidade das subescalas do instrumento foi verificada por meio da consistência interna com o cálculo do coeficiente alfa de Cronbach que considerou como limite inferior aceitável o valor de $0,50^{(7)}$. Para avaliar a correlação dos domínios com a questão 17 , foi utilizado o coeficiente de correlação de Spearman ${ }^{(8)}$.

A associação entre os valores dos domínios e as características gerais da amostra foi avaliada por meio do teste de Mann-Whitney (quando havia somente duas categorias) ou de Kruskal-Wallis (quando havia mais de duas categorias em cada variável). Para os testes estatísticos foi considerado o nível de significância de $5 \%$, isto é, p-valor $<0,05$.

\section{RESULTADOS}

A amostra foi composta por 88 pacientes em tratamento ambulatorial para HAS, com uma média de idade de 63,3 anos (dp $\pm 12,7)$, sendo que a maioria era do sexo feminino $(53,4 \%)$ e possuía o ensino fundamental incompleto $(62,4 \%)$.

O tabagismo foi referido por 5,7\% dos pacientes e o hábito de ingestão de bebida alcoólica foi verificado em 9,1\%. Dentre os que ingeriam bebida alcoólica, a cerveja foi referida por $62,5 \%$ dos pacientes. A prática de exercício físico foi verificada em $21,6 \%$ dos participantes, sendo a caminhada a atividade de escolha de $73,7 \%$. As demais características da amostra estão representadas na tabela 1 .

Tabela 1 - Características sociodemográficas e econômica de hipertensos em tratamento ambulatorial. Jundiaí, 2010

\begin{tabular}{lcc}
\hline \multicolumn{1}{c}{ Variáveis } & \multicolumn{2}{c}{ Resultados } \\
& n & \% \\
\hline Raça & 42 & 47,7 \\
Branca & 33 & 37,5 \\
Parda & 13 & 14,8 \\
Negra & & \\
Estado Civil & 43 & 48,8 \\
Casado & 23 & 26,1 \\
Viúvo & 7 & 8,0 \\
Solteiro & 7 & 8,0 \\
Separado & 6 & 6,8 \\
Divorciado & 2 & 2,3 \\
União Consensual & & \\
Renda Familiar & 44 & 50,0 \\
Um Salário Mínimo & 28 & 31,8 \\
Dois Salários Mínimos & 13 & 14,8 \\
Três Salários Mínimos & 3 & 3,4 \\
Quatro Salários ou Mais & 3 \\
\hline
\end{tabular}

Em relação ao tempo de HAS obteve-se que 52,3\% dos pacientes estavam em tratamento há mais de 10 anos. No que se refere às co-morbidades e complicações, as mais encontradas foram: angina $(61,4 \%)$, infarto agudo do miocárdio (39,8\%), diabetes $(37,5 \%)$ e acidente vascular encefálico (14,8\%).

No que se refere à análise do instrumento, os dados estão apresentados na tabela 2.

Tabela 2 - Média e desvio padrão das respostas de hipertensos em cada domínio do MINICHAL - BRASIL. Jundiaí, 2010

\begin{tabular}{lccc}
\hline \multicolumn{1}{c}{ Variável } & n & Média & Desvio-padrão \\
\hline Estado Mental & 88 & 7,5 & 4,9 \\
Manifestações & 88 & 4,8 & 3,4 \\
Somáticas & &
\end{tabular}

A correlação entre os domínios e a questão 17 ("Você diria que sua hipertensão e o tratamento dessa têm afetado sua qualidade de vida?"), resultou numa correlação positiva de moderada magnitude com ambos os domínios: estado mental $(\mathrm{r}=+0,40 ; \mathrm{p}<0,01)$ e manifestações somáticas $(r=+0,31 ; p<0,01)$.

Não foram encontradas diferenças significativas na associação entre os domínios e as diversas variáveis 
em estudo, como idade, sexo, raça, estado civil, renda familiar, escolaridade, tabagismo, etilismo, tempo de hipertensão e comorbidades prévias. A única associação significativa foi entre a subescala estado mental e a prática de exercício físico $(\mathrm{p}=0,02)$.

A análise da confiabilidade, calculada por meio do coeficiente alfa de Cronbach, foi de 0,74 para o domínio estado mental e 0,55 para o de manifestações somáticas, sendo considerada satisfatória.

\section{DISCUSSÃO}

Os participantes desse estudo obtiveram média de idade semelhante a encontrada por outros autores, nos quais se verificou que a HAS é mais comum em pessoas idosas ${ }^{(3)}$. Isso acontece porque os níveis tensionais tendem a se elevar em idades mais avançadas, devido ao desenvolvimento da aterosclerose, que leva a diminuição da complacência das artérias ${ }^{(1)}$.

O presente estudo detectou que a maioria dos participantes era do sexo feminino e possuía mais de 60 anos. Esses dados corroboram outro estudo que afirma que após essa idade a prevalência de HAS é maior nas mulheres ${ }^{(1)}$. No que se refere à raça, alguns autores, estudando hipertensão, também encontraram a maioria da amostra como sendo branca ${ }^{(5,9)}$, porém esses resultados diferem de outros que afirmam que a HAS é uma doença prevalente em indivíduos de cor não branca ${ }^{(1)}$.

A HAS, nesse estudo, também se mostrou mais prevalente entre os indivíduos com menor escolaridade e baixa renda; controle dos níveis pressóricos depende do acesso à saúde e educação e dessa forma, da capacidade do paciente em entender as orientações e aderir ao tratamento ${ }^{(1-2)}$.

Em relação ao hábito de fumar, os resultados diferem de outra pesquisa nacional que avaliou as atitudes e exposição de adultos ao tabagismo ${ }^{(10)}$. Um fato relevante no presente estudo é que muitos pacientes afirmaram que abandonaram o hábito de fumar antes da coleta de dados, porém não foi mensurado o tempo decorrido entre a data da coleta e o abandono do tabagismo. A Organização Mundial da Saúde considera o tabagismo o maior agente isolado e evitável de mortalidade e morbidade do mundo, sendo classificado como um dos principais fatores de risco para doença coronariana, hipertensão, acidente vascular encefálico, bronquite, enfisema e câncer, que são causas importantes de mortes no Brasil ${ }^{(10)}$.

Os resultados desta pesquisa são semelhantes aos obtidos por outros autores ao estudar o hábito de ingestão de bebida alcoólica. Na atualidade, o uso de álcool é caracterizado como um fator de risco para as doenças cardiovasculares e também um fenômeno social que representa um grave problema à saúde pública mundial, uma vez que o consumo abusivo acarreta inúmeras consequências negativas à qualidade de vida dos indivíduos e da população ${ }^{(11)}$.

A maioria dos hipertensos estudados não praticava atividade física. O sedentarismo também é considerado um fator de risco para as doenças cardiovasculares e para o tratamento da HAS recomenda-se a realização de, pelo menos, 30 minutos de atividade física, cinco vezes na semana. Os sujeitos, deste estudo, que praticavam atividade física davam preferência à caminhada, considerado o exercício aeróbio mais indicado aos hipertensos ${ }^{(2)}$.

No que se refere ao diagnóstico da doença, os pacientes desta pesquisa foram diagnosticados como hipertensos há mais tempo do que os estudados por outros autores ${ }^{(9)}$. Isso talvez possa ser explicado pelo fato da idade média dos portadores de HAS, deste estudo, também ser superior. Parcela considerável da amostra estudada referiu possuir co-morbidades e complicações da HAS (diabetes, angina, infarto, acidente vascular encefálico) cientificamente comprovadas por diversas literaturas ${ }^{(1-2,12)}$.

Ao comparar os resultados desta pesquisa com outra pesquisa nacional sobre qualidade de vida de hipertensos, as respostas dos sujeitos revelaram uma pior percepção de qualidade de vida tanto na subescala estado mental quanto na subescala manifestações somáticas ${ }^{(5)}$. Esses dados podem ter sido encontrados devido a amostra estudada apresentar uma média de idade superior ao do estudo citado e existir uma relação direta entre o envelhecimento e a piora da percepção de qualidade de vida ${ }^{(13)}$.

Outros autores $^{(14)}$, estudando qualidade de vida de pacientes diabéticos e hipertensos, também encontraram a mesma relação verificada neste estudo, ou seja, quanto melhor a percepção do paciente em relação à sua doença e o respectivo tratamento, melhor também é a sua avaliação nos domínios estado mental e manifestações somáticas. Pessoas que realizam atividade física possuem uma melhor percepção de qualidade de vida no domínio estado mental, pois sentem que estão adotando hábitos de vida saudáveis e conseqüentemente buscando o controle da $\mathrm{HAS}^{(15)}$.

\section{CONCLUSÃO}

A percepção de qualidade de vida de pacientes hipertensos submetidos a tratamento ambulatorial 
foi avaliada e mostrou-se inferior à de outros estudos devido às diferentes características da amostra.

A HAS é uma doença assintomática que somada à necessidade de mudanças de hábitos e permanente uso de medicação contribui para o comprometimento da qualidade de vida com conseqüente abandono do tratamento. Por isso, a realização da presente pesquisa contribui para que a equipe de saúde possa avaliar o impacto das intervenções propostas a fim de que se alcance o controle dos níveis pressóricos, sem comprometer a qualidade de vida de seus portadores.

Como existem poucos estudos, no Brasil, utilizando este instrumento, recomenda-se a realização de novas pesquisas que comparem a qualidade de vida dos portadores de hipertensão antes e após a implementação de intervenções, para que os resultados possam melhor auxiliar a equipe de saúde no planejamento da assistência.

\section{REFERÊNCIAS}

1. Sociedade Brasileira de Cardiologia, Sociedade Brasileira de Hipertensão, Sociedade Brasileira de Nefrologia. VI Diretrizes Brasileiras de Hipertensão Arterial. Arq. Bras. Cardiol. 2010;95(1 Supl 1):1-51.

2. Sociedade Brasileira de Cardiologia, Sociedade Brasileira de Hipertensão, Sociedade Brasileira de Nefrologia. V Diretrizes Brasileiras de Hipertensão Arterial. Arq. Bras. Cardiol. 2007;89(3):24-79.

3. Pinotti S, Mantovani MF, Giacomozzi LM. Percepção sobre hipertensão arterial e qualidade de vida: contribuição para o cuidado de enfermagem. Cogitare enferm. 2008;13(4):526-34.

4. The WHOQOL Group. The World Health Organization quality of life assessment (WHOQOL): position paper from the World Health Organization. Social science and medicin 1995;(10):1403-9.

5. Schulz RB, Rossignoli P, Correr CJ, Fernández-Limós F, Toni PM. Validação do mini-questionário de qualidade de vida em hipertensão arterial (MINICHAL) para o português (Brasil). Arq. Bras. Cardiol. 2008;90(2):139-44.

6. Ministério da Saúde (BR). Conselho Nacional de Saúde. Diretrizes e normas regulamentadoras de pesquisa envolvendo seres humanos. Resolução n. 196, de 10 de outubro de 1996. Brasília; 1996.

7. Bowling A. Measuring disease: a review of disease - specifc quality of life measuring scales. $2^{\text {a }}$ ed.Philadelphia: Open University Press; 2001.
8. Burns N, Grove SK. The practice of nursing research: conduct, critique \& utilization. Philadelphia: Saunders; 2001.

9. Melchiors AC, Correr CJ, Pantarolo R, Santos FOS, Souza RAP. Qualidade de vida em pacientes hipertensos e validade concorrente do MINICHAL-BRASIL. Arq. Bras. Cardiol. 2010;94(3):357-64.

10. Marcopito LF, Coutinho AP, Valencich DMO, Moraes MA, Brumini R, Ribeiro AS. Exposição ao tabagismo e atitudes: comparação entre inquéritos realizados na população adulta do município de São Paulo em 1987 e 2002. Arq. Bras. Cardiol.2007;89(5):333-40.

11. Vargas D, Oliveira MAF, Araujo EC. Prevalência de dependência alcoólica em serviços de atenção primária à saúde de Bebedouro, São Paulo, Brasil. Cad. Saúde Pública 2009;25(8):1711-20.

12. Carvalho MAN, Silva ISBS, Ramos SBP, Coelho LF, Gonçalves ID, Figueiredo Neto JA. Qualidade de vida de pacientes hipertensos e comparação entre dois instrumentos de medida de QVRS. Online Arq. bras. cardiol. [Internet] 2012; [acesso em 22 jun 2012]. Disponível: http://www.scielo.br/pdf/abc/2012nahead/ aop03112.pdf

13. Floriano PJ, Dalgalarrondo P. Saúde mental, qualidade de vida e religião em idosos de um Programa de Saúde da Família. J. bras. psiquiatr. 2007;56(3):162-70.

14. Miranzi SSC, Ferreira FS, Iwamoto HH, Pereira GA, Miranzi MAS. Qualidade de vida de indivíduos com diabetes mellitus e hipertensão acompanhados por uma equipe de saúde da família. Texto Contexto Enferm. 2008;17(4):672-9.

15. Spinato IL, Monteiro LZ, Santos ZMSA. Adesão da pessoa hipertensa ao exercício físico - uma proposta educativa em saúde. Texto Contexto Enferm. 2010;19(2):256-64. 